\title{
A Highly Tunable Silicone-Based Magnetic Elastomer with Nanoscale Homogeneity
}

\author{
Benjamin A. Evans ${ }^{a}{ }^{*}$, Briana L. Fiser ${ }^{b}$, Willem J. Prins ${ }^{a}$, Daniel J. Rapp ${ }^{a}$, Adam R. Shields ${ }^{c}$, \\ Daniel R. Glass ${ }^{a}$, and R. Superfine ${ }^{b}$ \\ aDepartment of Physics, Elon University, Elon, NC 27244 (USA) \\ bDepartment of Physics and Astronomy, University of North Carolina at Chapel Hill, Chapel Hill, \\ NC 27599 (USA) \\ ${ }^{\circ}$ Center for Bio/Molecular Science and Engineering, US Naval Research Laboratory, SW \\ Washington, DC, 20375 (USA)
}

\begin{abstract}
Magnetic elastomers have been widely pursued for sensing and actuation applications. Siliconebased magnetic elastomers have a number of advantages over other materials such as hydrogels, but aggregation of magnetic nanoparticles within silicones is difficult to prevent. Aggregation inherently limits the minimum size of fabricated structures and leads to non-uniform response from structure to structure. We have developed a novel material which is a complex of a silicone polymer (polydimethylsiloxane-co-aminopropylmethylsiloxane) adsorbed onto the surface of magnetite $\left(\gamma-\mathrm{Fe}_{2} \mathrm{O}_{3}\right)$ nanoparticles $7-10 \mathrm{~nm}$ in diameter. The material is homogenous at very small length scales $(<100 \mathrm{~nm})$ and can be crosslinked to form a flexible, magnetic material which is ideally suited for the fabrication of micro- to nanoscale magnetic actuators. The loading fraction of magnetic nanoparticles in the composite can be varied smoothly from $0-50 \%$ wt. without loss of homogeneity, providing a simple mechanism for tuning actuator response. We evaluate the material properties of the composite across a range of nanoparticle loading, and demonstrate a magnetic-field-induced increase in compressive modulus as high as $300 \%$. Furthermore, we implement a strategy for predicting the optimal nanoparticle loading for magnetic actuation applications, and show that our predictions correlate well with experimental findings.
\end{abstract}

\section{Keywords}

magnetic polymer; PDMS; composite materials; magnetic nanoparticles; actuators; microelectromechanical systems

\section{Introduction}

Stimuli-responsive micro- and nanoscale structures have many potential applications as sensors and actuators in the fields of microfluidics and microengineering. Of the various

\footnotetext{
(C) 2011 Elsevier B.V. All rights reserved

*Corresponding author at: Elon University, CB\# 2625, Elon, NC 27244, USA. Tel.: 13362786252. Fax: 3362786258. ben.evans@elon.edu..

Publisher's Disclaimer: This is a PDF file of an unedited manuscript that has been accepted for publication. As a service to our customers we are providing this early version of the manuscript. The manuscript will undergo copyediting, typesetting, and review of the resulting proof before it is published in its final citable form. Please note that during the production process errors may be discovered which could affect the content, and all legal disclaimers that apply to the journal pertain.
} 
strategies for actuating such structures, magnetic actuation has numerous advantages. For example, structures may be actuated by large-scale external fields, requiring no wire tethers to supply voltage or current, and magnetic fields are unlikely to interfere with sensitive chemical reactions. Thus, much recent work has been devoted to the development of microscale structures capable of magnetic actuation. Currently, most magnetically actuated microstructures consist of thin magnetic components bound to flexible substrates[1;2;3;4; 5] or paramagnetic chains formed by linking colloidal particles[6;7]. However, another class of magnetic microactuator is emerging in which devices are composed of a monolithic material which is both flexible and magnetic[8; 9]. A chief difficulty in engineering a monolithic magnetic actuator lies in designing the properties of the material itself to achieve an optimal magnetic response. The responsiveness of a structure is a competition between flexibility and magnetic permeability, properties which are generally mutually exclusive in pure materials. Thus, these properties must be engineered into a composite material, such as a composite of highly permeable magnetic nanoparticles in a flexible polymer matrix.

Magnetic nanoparticles generally mix easily into aqueous suspensions, and so the most common choices for polymer networks by far are soft hydrogels such as those constructed of polyacrylamide[10; 11; 12], N-isopropylacrylamide[13], polyvinyl alcohol[14; 15; 16;17; 18], and their derivative copolymers[19;20]. Also prevalent are gelatin[21] and copolymers of polyethylene oxide[22; 23]. Hydrophobic matrices are much less common, but are preferable in many instances since they are generally chemically and osmotically inert in aqueous environments. However, obtaining a homogenous dispersion of magnetic micro- or nanoparticles in a hydrophobic matrix such as silicone is challenging. While numerous instances of iron particles dispersed in a silicone elastomer have been reported, very little attention has been given to the microscale homogeneity of these materials which is so critical to their implementation in microactuator applications. In most cases, the magnetic component consists of $2-4$ um carbonyl iron particles[24; 25; 26; 27] or commerciallyavailable iron powders containing particles $200 \mathrm{~nm}$ or larger[25; 27], and the iron particles are simply mixed into the uncrosslinked silicone, occasionally with the aid of a surfactant. The large size of the particles, compounded by the tendency of iron oxide particles to aggregate in a silicone matrix, generally renders these materials unsuitable for microactuator applications. Large particles and particle aggregation severely limit the size of structures which can be fabricated and introduce sources of inhomogeneity in actuator response from structure to structure.

We are aware of only two reports of magnetic silicone elastomers containing magnetic particles smaller than $100 \mathrm{~nm}$. In one instance, researchers note aggregates in materials with particle loadings greater than $0.1 \%$ wt.[28]; the second is a prior report from our group[8]. In both cases, the nanoparticles are merely entrapped within the polymer network. In the ideal material, silicone polymer would be adsorbed directly onto the surface of the magnetic nanoparticles. The resulting core-shell nanostructure would itself be useful in a variety of applications ranging from biocompatible magnetic hyperthermia agents to environmental toxin absorbents[29]; more notably, such processing would entirely eliminate the potential for aggregation or leaching of nanoparticles in the crosslinked bulk.

We note that in recent years researchers have succeeded in producing a just such a material by complexing a functionalized polydimethylsiloxane (PDMS) with cobalt and iron oxide nanoparticles[30; 31]. However, to our knowledge this material has not been crosslinked to form a solid. In this work therefore, we describe the first instance of a magnetic silicone elastomer in which a functionalized silicone is absorbed directly onto the surface of iron oxide nanoparticles $(7-10 \mathrm{~nm})$. The presence of the polymer surrounding each individual nanoparticle eliminates the potential for aggregation, resulting in a composite material with 
unprecedented uniformity at the nanoscale. Furthermore, iron nanoparticle content can be varied smoothly from $0-50 \%$ wt. without any loss of homogeneity.

The responsiveness of any magnetic actuator is the result of a competition between magnetic and elastic properties: a higher magnetic permeability enables a greater magnetic torque; however, the very nanoparticles which contribute toward permeability also increase the modulus of the material, rendering it less flexible. Thus, we characterize both the magnetic and elastic properties of this material across the full range of nanoparticle loadings. We measure elastic modulus both in the presence and absence of a moderate $300 \mathrm{mT}$ magnetic field, and find a dramatic field-induced increase in elastic modulus at higher nanoparticle loadings. This sharp increase in modulus at higher loadings suggests that there exists an optimal magnetite loading at which the material is most responsive to magnetic actuation. We demonstrate this optimization by constructing and actuating microstructures of this new composite material across a range of nanoparticle loadings and show that our experimental data correlates well with predictions based on established models.

\section{Experimental}

\subsection{Materials}

Ferric chloride $\left(\mathrm{FeCl}_{3}\right)$, ferrous chloride tetrahydrate $\left(\mathrm{FeCl}_{2}+4 \mathrm{H}_{2} \mathrm{O}\right)$, and ammonium hydroxide were obtained from Sigma Aldrich. Dicumyl peroxide was obtained from Fisher Scientific. (Tridecafluoro-1,1,2,2-tetrahydrooctyl) trichlorosilane (SIT8174.0) and the copolymer of aminopropylmethylsiloxane (APMS) with dimethylsiloxane (DMS), with 6-7 percent APMS mole \% (AMS-161) were obtained commercially from Gelest, Inc. All were used as received.

\subsection{Synthesis}

Following the work of Massart[32; 33] and van Ewijk[34], the magnetite nanoparticles used in this work are precipitated from ferric chloride $\left(\mathrm{FeCl}_{3}\right)$ and ferrous chloride $\left(\mathrm{FeCl}_{2}\right)$ salts in a 2:1 molar concentration. Ferric chloride $\left(\mathrm{FeCl}_{3}, 1.2 \mathrm{~g}\right)$ and ferrous chloride tetrahydrate $\left(\mathrm{FeCl}_{2}+4 \mathrm{H}_{2} \mathrm{O}, 0.74 \mathrm{~g}\right)$ are each dissolved in deionized water $(20 \mathrm{~mL})$ and then mixed together and stirred for several minutes. While stirring vigorously, concentrated ammonium hydroxide ( $20 \mathrm{~mL}, 29 \% \mathrm{wt}$. in water) is added to precipitate the salts and form magnetite $\left(\mathrm{Fe}_{2} \mathrm{O}_{3}\right)$ nanoparticles.

We complex these nanoparticles with a copolymer of aminopropylmethylsiloxane (APMS) and dimethylsiloxane (DMS), containing 6-7 mole \% APMS. For simplicity, we will refer to this polymer as PDMS- $\mathrm{NH}_{2}$. The APMS segment of the PDMS- $\mathrm{NH}_{2}$ contains amine groups which adsorb onto the surface of the magnetite nanoparticles under appropriate conditions, but the segments are short enough $(\sim 0.6 \mathrm{~nm})$ to be unlikely to bind multiple particles. By tuning the $\mathrm{pH}$ of the aqueous solution containing the magnetite nanoparticles to be greater than the isoelectric point of magnetite $(\mathrm{pH} 6.8)$ [35] and less than the $\mathrm{pKa}$ of the secondary amines $(\mathrm{pH} \sim 10)$, conditions can be created in which the amine functionality of the siloxane copolymer will bind to the positively-charged magnetite particle, transferring the magnetite from the aqueous phase (nanoparticles in water) to the organic phase (PDMS$\mathrm{NH}_{2}$ ) and yielding a siloxane-magnetite complex. We find that this occurs sufficiently well at the unadjusted $\mathrm{pH}$ of the nanoparticle solution following precipitation. After stirring the nanoparticle solution for several minutes, PDMS- $\mathrm{NH}_{2}(2 \mathrm{~mL})$ is added, and the mixture is stirred vigorously for 24 hours, during which time it will separate into two phases: a light-toclear aqueous phase and a thick, black organic phase.

Since the resulting complex is a ferrofluid (FF), we refer to it as FFPDMS-NH $\mathrm{N}_{2}$. A diagram detailing the synthesis of FFPDMS- $\mathrm{NH}_{2}$ is shown in Figure 1. Scanning electron 
microscopy clearly shows a uniform distribution of iron oxide nanoparticles embedded in a polymer matrix, indicating remarkable homogeneity at the microscale.

The organic phase consists largely of the magnetite-PDMS- $\mathrm{NH}_{2}$ complex, but also contains some amount of uncomplexed magnetite, uncomplexed PDMS-NH $\mathrm{NH}_{2}$, and entrained water and ammonia. To separate the complexed material from the byproducts, the aqueous phase is decanted and the PDMS phase is rinsed copiously with methanol and mechanical stirring to remove excess uncomplexed PDMS- $\mathrm{NH}_{2}$. The resulting suspension is sedimented with a permanent magnet, and the methanol is decanted.

The sediment is rinsed five times in methanol, five times in water, and five times again in methanol, sedimenting with a magnet and decanting each time. Finally, we add $15 \mathrm{~mL}$ of chloroform and the mixture is sonicated in a bath ultrasonicator for 30 minutes. At this point the complexed material forms a stable suspension in the chloroform, resulting in an extremely dark, unclouded solution. The solution is allowed to sediment overnight on a permanent magnet to remove any uncomplexed magnetite aggregates. Magnetite concentration may be tuned at this point by diluting the suspension with additional PDMS$\mathrm{NH}_{2}$ followed by 30 minutes of ultrasonication prior to removing the solvent. In this manner, we were able to adjust the magnetic content of the composite smoothly from $0 \%$ to $50 \%$ wt. iron without any particle aggregation. Observation of microstructures made of FFPDMS- $\mathrm{NH}_{2}$ dilutions by optical microscopy with a $40 \times$ objective yield no visible inhomogeneities in the material, and TEM imaging indicates that particles are welldispersed even in crosslinked materials (Figure 2).

Crosslinking may be accomplished by adding $10 \%$ wt. dicumyl peroxide to the FFPDMS$\mathrm{NH}_{2}$. After adding the peroxide, the mixture is stable at room temperature for several months and will cure in approximately 2 hours at $180^{\circ} \mathrm{C}$. A pre-treatment at $80^{\circ} \mathrm{C}$ for two hours will prevent bubbles from forming during the crosslinking step.

\subsection{Characterization}

In this work, we measured the elastic modulus and magnetization of $20 \%-90 \%$ wt. nominal dilutions of FFPDMS-NH $\mathrm{NH}_{2}$ complex into PDMS-NH $\mathrm{NH}_{2}$. These dilutions correspond to $9 \%-$ $50 \%$ wt. concentrations of magnetite nanoparticles (Table 1). In addition, we constructed microactuators of each dilution and quantified the bending of each in a $300 \mathrm{mT}$ magnetic field. This field magnitude is typical near the surface of a neodymium iron boride magnet, and is thus relevant to many microscale actuation systems.

2.3.1 Young's Modulus-Each material was poured into a cylindrical polydimethylsiloxane (PDMS) mold $(2.5 \mathrm{~mm}$ diam. $\times 3 \mathrm{~mm}$ length $)$ and cured at $180^{\circ} \mathrm{C}$ for 2-6 hours. To prevent the mold and material from adhering to one another, the PDMS mold was pretreated with a release agent via vapor deposition: the mold was plasma-treated for 20 seconds and then placed in a vacuum desiccator with (tridecafluoro-1,1,2,2-tetrahydrooctyl) trichlorosilane $(200 \mathrm{uL})$ for one hour prior to filling with FFPDMS-NH $\mathrm{N}_{2}$.

Young's modulus measurements of the cured samples were taken with an in-house modulometer constructed of a piezoelectric force sensor and micrometer. Measurements were taken under compression and in the low strain region $(<10 \%$ strain) of multiple stressstrain curves for each material, since low strain is characteristic of high-aspect-ratio actuators. Moduli were measured for each sample first in ambient conditions and then under the influence of a uniform $300 \mathrm{mT}$ magnetic field provided by a neodymium iron boride magnet and oriented parallel to the direction of compression. The variation in the field magnitude over the sample space was less than $10 \mathrm{mT}$. As shown in Figure 3, we found that modulus was linearly dependent on magnetic loading in the absence of a magnetic field, but 
that under the influence of a $300 \mathrm{mT}$ field modulus increased precipitously for higher magnetite loadings. Modulus measurements are presented in both Table 1 and Figure 3.

2.3.2 Magnetization-We used SQUID magnetometry (Quantum Design Magnetic Property Measurement Systems) to obtain a full magnetization curve for magnetite nanoparticles and saturation magnetizations for each of the dilutions. The magnetization of magnetite nanoparticles was measured while varying the field between -5 and $+5 \mathrm{~T}$ at room temperature $(300 \mathrm{~K})$. Saturation magnetization values for crosslinked FFPDMS- $\mathrm{NH}_{2}$ samples of varying magnetic concentrations were taken at fields approaching $5 \mathrm{~T}$.

No significant hysteresis is observed, indicating that the material is superparamagnetic as expected. This is a desirable feature in a magnetic actuator, since it avoids complications due to remanence magnetization. As shown in Figure 4, the saturation magnetization increases linearly with the \% wt. of FFPDMS- $\mathrm{NH}_{2}$ in $\mathrm{PDMS}-\mathrm{NH}_{2}$. The saturation magnetizations fall well within the range of previously reported saturation values for magnetite and maghemite polymer complexes[31;36;37;38], which range as high as 50 $\mathrm{Am}^{2} \mathrm{~kg}^{-1}$.

By extrapolation of the linear fit to the saturation magnetization data in Figure 4, we can predict that a $100 \%$ FFPDMS- $\mathrm{NH}_{2}$ sample would have a mass magnetization of $29.1 \mathrm{Am}^{2}$ / $\mathrm{kg}$. We have measured the saturation magnetization of the uncoated magnetite nanoparticles used in this work to be $51.5 \mathrm{Am}^{2} / \mathrm{kg}$, and can therefore determine that a $100 \%$ sample (the complex itself with no excess polymer) would consist of $56.5 \%$ wt. ( $21.1 \%$ vol.) magnetite. A similar extrapolation of the density data in Figure 4 yields the same result to within a $2 \%$ relative difference. Such volume loading corresponds to a complex radius which is 1.7 times larger than the radius of the nanoparticle core, or an average polymer thickness of approximately $3 \mathrm{~nm}$ for our $7-10 \mathrm{~nm}$-diameter magnetite nanoparticles. This number is not inconsistent with a polymer monolayer.

2.3.3 Microactuators-In addition to bulk samples, high-aspect-ratio microstructures (75 $\mu \mathrm{m} \times 2.5 \mathrm{~mm}$ ) of each dilution were fabricated and actuated with a spatially-uniform 300 $\mathrm{mT}$ magnetic field. To produce high-aspect-ratio FFPDMS- $\mathrm{NH}_{2}$ microstructures $(75 \mu \mathrm{m} \times$ $2.5 \mathrm{~mm}$ ), $33 \mathrm{G}$ needles were used to drill $210-\mu \mathrm{m}$ holes in a pre-strained polystyrene (Shrinky-Dinks, goestores.com, item\# D700-6A) which relaxed to its unstrained dimensions upon heating to $180^{\circ} \mathrm{C}$. FFPDMS- $\mathrm{NH}_{2}$ with dicumyl peroxide was pressed into the resulting $75 \mu \mathrm{m}$ diameter $\times 2.5 \mathrm{~mm}$ pores and cured at $180^{\circ} \mathrm{C}$ for $2-6$ hours, after which the polystyrene template was dissolved in chloroform. The FFPDMS- $\mathrm{NH}_{2}$ cylinders were then affixed to a glass substrate and positioned between the poles of an electromagnet (Atomic Laboratories, cat. \# 79641) configured to produce a field magnitudes of $300 \mathrm{mT}$ with very low magnetic field gradients $(<5 \mathrm{G} / \mathrm{mm})$. All structures were positioned such that their initial orientation was $70^{\circ}$ out of alignment with the magnetic field and were actuated across a range of field magnitudes, up to and including $300 \mathrm{mT}$. Images were taken with a digital camera mounted on a dissection scope and bending angles were calculated from analysis of the tip displacement.

It is important to note that a high-aspect-ratio magnetic microstructure may, in general, actuate by two distinct mechanisms: 1) a torque caused by the misalignment of the long axis of the microstructure with a magnetic field, and 2) a force caused by a magnetic field gradient. In most microactuator applications, the former is dominant [8;39]. Therefore the actuating field in this experiment was designed with a minimal gradient to ensure that actuation was well within the field-dominated regime. To verify that this is the case, the ratio of 'gradient' torque to 'field' torque should be significantly less than unity. This ratio is given by $R=2 \nabla \mathrm{BL} / \mu_{0} M \rho$, where $L$ is the length of the actuator, $M$ is the mass 
magnetization of the composite, and $\rho$ is the density[39]. In our experiments, $R$ ranges from 0.25 to 0.027 , indicating dominance of the field-mediated mechanism.

\section{Results and Discussion}

A material which is intended for use as an actuator must be both flexible and have a large magnetic permeability. However, adding high-modulus magnetic particles to a composite to achieve higher magnetic permeability generally increases the modulus of the composite, resulting in a less flexible material. A method for determining the optimal loading of magnetic particles which will result in the most responsive material is therefore critical. The responsiveness of a material in this sense is quantified by a figure of merit which has been proposed in various forms by several previous studies [39; 40; 41; 42]. In brief, the angle through which a cantilevered high-aspect-ratio magnetic microstructure is expected to bend is given by the following[39], in which $M$ is the mass magnetization of the composite material, $\rho$ is the density, $E$ is the Young's modulus, and $L / 2 r$ is the aspect ratio of the actuator:

$$
\theta=\frac{\mu_{0} M^{2} \rho^{2}}{E}\left(\frac{L}{2 r}\right)^{2}
$$

The first term in this expression represents the influence of material properties and is independent of geometrical considerations. As such, it is extremely useful in the design of materials for effective actuators. Since this term represents the material-dependent component of the ratio of magnetic to elastic torques on a high-aspect-ratio microactuator, we refer to it as the magnetoelastic ratio. A higher magnetoelastic ratio indicates a material that will bend more easily in response to a uniform applied magnetic field, regardless of geometrical configuration. It should be noted that the magnetization $M$ is in general a function of applied field. In our experiments with FFPDMS- $\mathrm{NH}_{2}$, we assume that magnetization is proportional to the loading fraction of magnetic nanoparticles and we may therefore use the full the magnetization curve of the magnetite nanoparticles (Figure 4A) to deduce the mass magnetization of each sample at $300 \mathrm{mT}$.

Magnetoelastic ratios for each of the dilutions of FFPDMS- $\mathrm{NH}_{2}$ were calculated 1) from measurements of modulus, mass magnetization and density and 2) from microactuator bending experiments. The former are shown in Table 1 and both sets of calculations are plotted in Figure 5. In both cases, the data show a clear peak in magnetoelastic ratio around a 60\%-70\% nominal concentration of FFPDMS-NH $\mathrm{NH}_{2}$ in $\mathrm{PDS}-\mathrm{NH}_{2}$, which represents a total \% wt. loading of magnetite nanoparticles of approximately $37-39 \%$. Materials with lower magnetic content are less responsive to the magnetic field; materials with higher magnetic content are too stiff to bend sufficiently.

It should be noted that the sharp increase in modulus with particle loading exhibited in Figure 3 is critical in producing the peak in the magnetoelastic ratio within the range of samples studied in this work. Field-induced increases in moduli of iron / polymer composites have been reported previously[24; 26; 27; 43; 44; 45], and several works develop models which predict the dependence of elastic or shear moduli on magnetic field in such materials. Some report on the shear modulus of parallel chains of nanoparticles embedded in a soft matrix, such as may be produced by curing the matrix in the presence of a magnetic field[24; 43]. These predict a linear dependence on nanoparticle loading fraction; however, it is clear that these models cannot be applied to an isotropic material. Later models do address the compressive modulus of isotropic dispersions of magnetic particles in a matrix. However, while these models show how modulus depends on magnetic field 
strength[27] or the degree of magnetization relative to saturation[46], in both cases the dependence on loading fraction, $\varphi$, is buried within fitting parameters. Thus we have found no model which predicts the dependence of modulus on the loading fraction of magnetic nanoparticles in an isotropic composite. In this work, we find that the increase in modulus caused by the applied field is best fit by a function of the form $\Delta E(\varphi)=\alpha \exp ^{\beta \varphi}$, as shown in Figure 3B.

Furthermore, while some studies have reported moduli of composites of varying particle loading in a magnetic field, the results from one study to the next vary widely. For example, Filipcsei et al. report a modest $20 \%$ increase in elastic modulus for a $30 \% \mathrm{wt}$. isotropic composite of 200-nm magnetite in a silicone[27], while Stepanov et al. report a 10-fold increase in modulus for a $35 \%$ wt. isotropic composite of 2-70 um iron particles in a silicone of similar zero-field modulus[44]. We suspect that much of this variation may be due to inhomogeneities in the materials caused by aggregation effects, or to a broad disparity of particle sizes within the material. The complex presented in this work may therefore constitute an ideal platform for experimental inquiry into the effect of particle loading on modulus in magnetic elastomers.

Since this material is of interest to the magnetic microactuation community, we compare our material to existing magnetic / polymer composite materials in Figure 6, which is adapted from Evans et al., 2011[39]. We restrict this plot to materials with magnetic particles smaller than $100 \mathrm{~nm}$, since larger particles are not ideal for microactuator applications. In addition, we broadly segregate magnetic composite materials into the two most commonly-reported classes: hydrogels and silicones. The utility of each class depends largely on the specific application under consideration: while hydrogels have much lower moduli, silicones have the advantage of being chemically and osmotically inert in aqueous environments and stable when exposed to atmosphere. In the silicone class, our material has the highest magnetoelastic ratio of available composites with particle sizes smaller than 100 microns.

\section{Conclusions}

We have produced a novel material consisting of a homogenous dispersion of complexed magnetic nanoparticles within a flexible silicone copolymer. When crosslinked, this material is both flexible and magnetic, introducing new possibilities for monolithic micromagnetomechanical systems. We have demonstrated that the material has a clear optimal configuration for magnetic actuation applications which can be determined simply by measuring its mechanical and magnetic properties. As the field of microscale actuators expands, we expect this strategy will be a useful tool in designing materials.

A curable magnetic/polymer composite material such as this may find use in many applications, ranging from scalable microbead technology to microactuators. With this material, we have produced our own actuator arrays, which consist of high-aspect ratio cantilevered microrods roughly the size of human lung cilia, similar to those previously reported[8], which will be presented in a future publication. This system has been magnetically actuated in a fluid and serves to demonstrate the utility of this new material in actuator applications. Furthermore, we expect that the unique microscale homogeneity of this silicone composite may be of use in exploring the fundamental physics responsible for magnetically-enhanced modulus in magnetic elastomers.

\section{Acknowledgments}

This work was funded in part by NSF NIRT (CMS-0507151), the Virtual Lung Project (NIH R01HL077546-01A2), the Center for Computer Integrated Systems for Microscopy and Manipulation (NIH P41EB002025-24A1), and the Office of Undergraduate Research at Elon University. 


\section{References}

[1]. Judy JW, Muller RS. Magnetic microactuation of torsional polysilicon structures. Sensors and Actuators a-Physical. 1996; 53:392-397.

[2]. Judy JW, Muller RS, Zappe HH. Magnetic microactuation of polysilicon flexure structures. Journal of Microelectromechanical Systems. 1995; 4:162-169.

[3]. Khoo M, Liu C. Micro magnetic silicone elastomer membrane actuator. Sensors and Actuators aPhysical. 2001; 89:259-266.

[4]. Sadler DJ, Liakopoulos TM, Ahn CH. A universal electromagnetic microactuator using magnetic interconnection concepts. Journal of Microelectromechanical Systems. 2000; 9:460-468.

[5]. Liu C. Development of surface micromachined magnetic actuators using electroplated permalloy. Mechatronics. 1998; 8:613-633.

[6]. Singh H, Laibinis PE, Hatton TA. Synthesis of flexible magnetic nanowires of permanently linked core-shell magnetic beads tethered to a glass surface patterned by microcontact printing. Nano Letters. 2005; 5:2149-2154. [PubMed: 16277443]

[7]. Furst EM, Suzuki C, Fermigier M, Gast AP. Permanently linked monodisperse paramagnetic chains. Langmuir. 1998; 14:7334-7336.

[8]. Evans BA, Shields AR, Carroll RL, Washburn S, Falvo MR, Superfine R. Magnetically actuated nanorod arrays as biomimetic cilia. Nano Letters. 2007; 7:1428-1434. [PubMed: 17419660]

[9]. Shields AR, Fiser BL, Evans BA, Falvo MR, Washburn S, Superfine R. Biomimetic cilia arrays generate simultaneous pumping and mixing regimes. Proceedings of the National Academy of Sciences of the United States of America. 2010; 107:15670-15675. [PubMed: 20798342]

[10]. Caykara T, Yoruk D, Demirci S. Preparation and Characterization of Poly(Ntertbutylacrylamide-co-acrylamide) Ferrogel. Journal of Applied Polymer Science. 2009; 112:800-804.

[11]. Galicia JA, Sandre O, Cousin F, Guemghar D, Menager C, Cabuil V. Designing magnetic composite materials using aqueous magnetic fluids. Journal of Physics-Condensed Matter. 2003; 15:S1379-S1402.

[12]. Mayer CR, Cabuil V, Lalot T, Thouvenot R. Magnetic nanoparticles trapped in pH 7 hydrogels as a tool to characterize the properties of the polymeric network. Advanced Materials. 2000; 12:417-+.

[13]. Xulu PM, Filipcsei G, Zrinyi M. Preparation and responsive properties of magnetically soft poly(N-isopropylacrylamide) gels. Macromolecules. 2000; 33:1716-1719.

[14]. Barsi, L.; Buki, A.; Szabo, D.; Zrinyi, M. Gels with Magnetic Properties. Progress in Colloid and Polymer Science, Springer; Berlin: 1996. p. 57-63.

[15]. Collin D, Auernhammer GK, Gavat O, Martinoty P, Brand HR. Frozen-in magnetic order in uniaxial magnetic gels: Preparation and physical properties. Macromolecular Rapid Communications. 2003; 24:737-741.

[16]. Lin H, Watanabe Y, Kimura M, Hanabusa K, Shirai H. Preparation of magnetic poly(vinyl alcohol) (PVA) materials by in situ synthesis of magnetite in a PVA matrix. Journal of Applied Polymer Science. 2003; 87:1239-1247.

[17]. Mitsumata T, Ikeda K, Gong JP, Osada Y, Szabo D, Zrinyi M. Magnetism and compressive modulus of magnetic fluid containing gels. Journal of Applied Physics. 1999; 85:8451-8455.

[18]. Resendiz-Hernandez, PJ.; Rodriguez-Fernandez, OS.; Garcia-Cerda, LA. Synthesis of poly(vinyl alcohol)-magnetite ferrogel obtained by freezing-thawing technique. Elsevier Science Bv; 2008. p. E373-E376.

[19]. Lin CL, Chiu WY, Don TM. Superparamagnetic thermoresponsive composite latex via W/O miniemulsion polymerization. Journal of Applied Polymer Science. 2006; 100:3987-3996.

[20]. Fuhrer R, Athanassiou EK, Luechinger NA, Stark WJ. Crosslinking Metal Nanoparticles into the Polymer Backbone of Hydrogels Enables Preparation of Soft, Magnetic Field-Driven Actuators with Muscle-Like Flexibility. Small. 2009; 5:383-388. [PubMed: 19180549]

[21]. Saslawski O, Weingarten C, Benoit JP, Couvreur P. MAGNETICALLY RESPONSIVE MICROSPHERES FOR THE PULSED DELIVERY OF INSULIN. Life Sciences. 1988; 42:1521-1528. [PubMed: 3280940] 
[22]. Qin J, Asempah I, Laurent S, Fornara A, Muller RN, Muhammed M. Injectable Superparamagnetic Ferrogels for Controlled Release of Hydrophobic Drugs. Advanced Materials. 2009; 21:1354-1357.

[23]. Wormuth K. Superparamagnetic latex via inverse emulsion polymerization. Journal of Colloid and Interface Science. 2001; 241:366-377.

[24]. Jolly MR, Carlson JD, Munoz BC, Bullions TA. The magnetoviscoelastic response of elastomer composites consisting of ferrous particles embedded in a polymer matrix. Journal of Intelligent Material Systems and Structures. 1996; 7:613-622.

[25]. Abramchuk SS, Grishin DA, Kramarenko EY, Stepanov GV, Khokhlov AR. Effect of a homogeneous magnetic field on the mechanical behavior of soft magnetic elastomers under compression. Polymer Science Series A. 2006; 48:138-145.

[26]. Farshad M, Benine A. Magnetoactive elastomer composites. Polymer Testing. 2004; 23:347-353.

[27]. Filipcsei, G.; Csetneki, I.; Szilagyi, A.; Zrinyi, M. Magnetic field-responsive smart polymer composites. Oligomers Polymer Composties Molecular Imprinting, Springer-Verlag; Berlin, Berlin: 2007. p. 137-189.

[28]. Fahrni F, Prins MWJ, van IJzendoorn LJ. Magnetization and actuation of polymeric microstructures with magnetic nanoparticles for application in microfluidics. Journal of Magnetism and Magnetic Materials. 2009; 321:1843-1850.

[29]. Darling SB, Bader SD. A materials chemistry perspective on nanomagnetism. Journal of Materials Chemistry. 2005; 15:4189-4195.

[30]. Mefford OT, Carroll MRJ, Vadala ML, Goff JD, Mejia-Ariza R, Saunders M, Woodward RC, Pierre TGS, Davis RM, Riffle JS. Size analysis of PDMS-magnetite nanoparticle complexes: Experiment and theory. Chemistry of Materials. 2008; 20:2184-2191.

[31]. Vadala ML, Rutnakornpituk M, Zalich MA, St Pierre TG, Riffle JS. Block copolysiloxanes and their complexation with cobalt nanoparticles. Polymer. 2004; 45:7449-7461.

[32]. Massart R. Preparation of Aqueous Magnetic Liquids in Alkaline and Acidic Media. IEEE Transactions on Magnetics. 1981; 17:1247-1248.

[33]. Massart R, Dubois E, Cabuil V, Hasmonay E. Preparation and Properties of Monodisperse Magnetic Fluids. Journal of Magnetism and Magnetic Materials. 1995; 149:1-5.

[34]. van Ewijk GA, Vroege GJ, Philipse AP. Convenient preparation methods for magnetic colloids. Journal of Magnetism and Magnetic Materials. 1999; 201:31-33.

[35]. Tewari PH, Mclean AW. Temperature Dependence of Point of Zero Charge of Alumina and Magnetite. Journal of Colloid and Interface Science. 1972; 40:267-\&.

[36]. Francois NJ, Allo S, Jacobo SE, Daraio ME. Composites of polymeric gels and magnetic nanoparticles: Preparation and drug release behavior. Journal of Applied Polymer Science. 2007; 105:647-655.

[37]. Kryszewski M, Jeszka JK. Nanostructured conducting polymer composites - superparamagnetic particles in conducting polymers. Synthetic Metals. 1998; 94:99-104.

[38]. Wilson KS, Harris LA, Goff JD, Riffle JS, Dailey JP. A generalized method for magnetite nanoparticle stericc stabilization utilizing block copolymers containing carboxylic acids. European Cells and Materials. 2002; 3:206-209.

[39]. Evans, BA.; Superfine, R. Design Considerations for Magnetically Actuated Biomimetic Cilia. In: George, A., editor. Biomimetic Based Applications. InTech; Rijeka, Croatia: 2011.

[40]. Roper M, Dreyfus R, Baudry J, Fermigier M, Bibette J, Stone HA. On the dynamics of magnetically driven elastic filaments. Journal of Fluid Mechanics. 2006; 554:167-190.

[41]. Cebers A, Javaitis I. Bending of flexible magnetic rods. Physical Review E. 2004; 70

[42]. Gauger EM, Downton MT, Stark H. Fluid transport at low Reynolds number with magnetically actuated artificial cilia. European Physical Journal E. 2009; 28:231-242.

[43]. Shen Y, Golnaraghi MF, Heppler GR. Experimental research and modeling of magnetorheological elastomers. Journal of Intelligent Material Systems and Structures. 2004; 15:27-35. 
[44]. Stepanov GV, Abramchuk SS, Grishin DA, Nikitin LV, Kramarenko EY, Khokhlov AR. Effect of a homogeneous magnetic field on the viscoelastic behavior of magnetic elastomers. Polymer. 2007; 48:488-495.

[45]. Papaphilippou PC, Pourgouris A, Marinica O, Taculescu A, Athanasopoulos GI, Vekas L, Krasia-Christoforou T. Fabrication and characterization of superparamagnetic and thermoresponsive hydrogels based on oleic-acid-coated Fe3O4 nanoparticles, hexa(ethylene glycol) methyl ether methacrylate and 2-(acetoacetoxy)ethyl methacrylate. Journal of Magnetism and Magnetic Materials. 2011; 323:557-563.

[46]. Wood DS, Camp PJ. Modeling the properties of ferrogels in uniform magnetic fields. Physical Review E. 2011; 83:9. 


\section{Highlights}

$>$ A silicone-magnetite elastomer with nanoscale homogeneity.

$>\quad$ Iron content tunable from $0-50 \%$ wt. without aggregation.

$>\quad$ Elastic modulus increases in presence of magnetic field.

$>\quad$ Model and experiment show maximal actuation for microstructures near $40 \%$ wt. iron. 


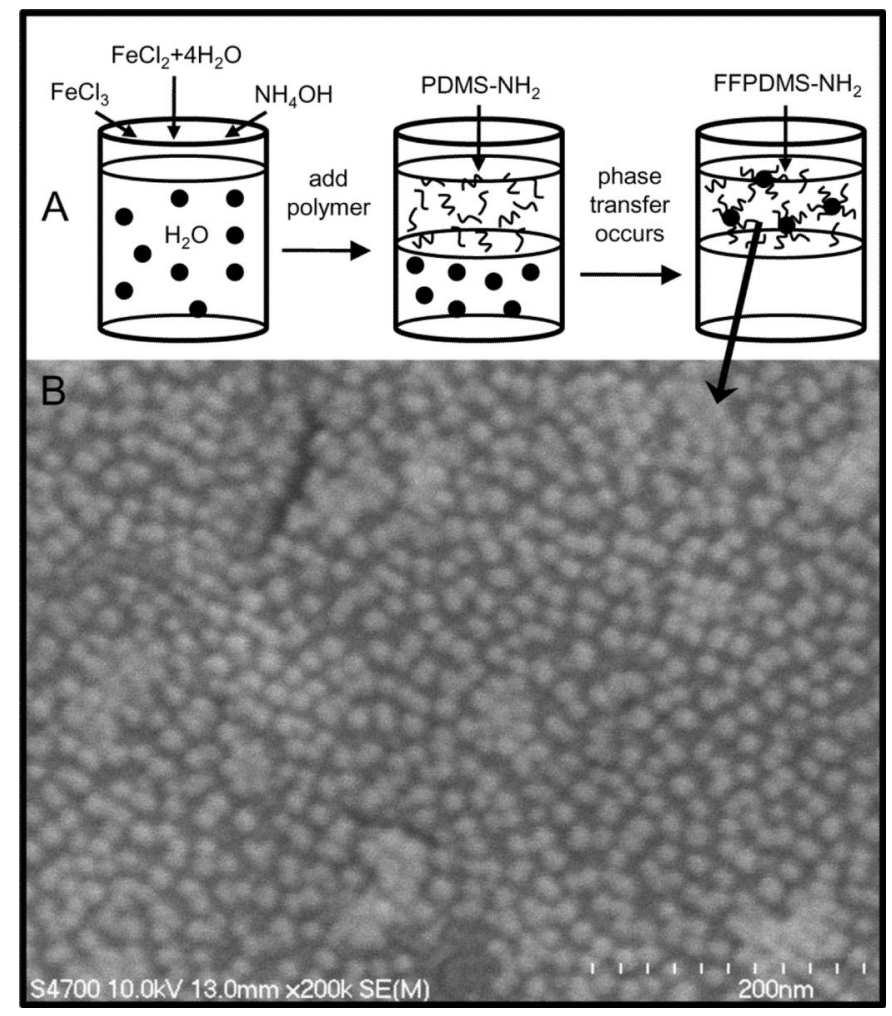

Fig. 1.

A) Synthesis of FFPDMS-NH . Iron oxide nanoparticles are precipitated from iron salts upon the addition of ammonium hydroxide. $\mathrm{PDMS}-\mathrm{NH}_{2}$ is then added to the nanoparticle solution, and the mixture is left to stir overnight. The nanoparticles move into the organic phase due to their electrostatic attraction to the PDMS-NH $\mathrm{NH}_{2}$, yielding FFPDMS-NH 2. B) Scanning electron micrograph of a film of uncrosslinked FFPDMS- $\mathrm{NH}_{2}$ in which individual nanoparticles are clearly visible. The material is homogeneous at small length scales. 


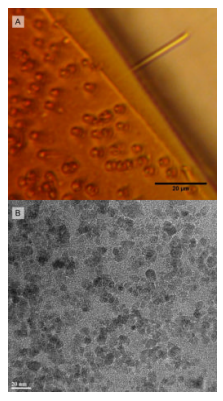

Fig. 2.

A) Cylindrical microstructures $(2 \mu \mathrm{m}$ diam. $\times 25 \mu \mathrm{m}$ length) constructed of a $60 \%$ FFPDM$\mathrm{SNH}_{2}$. In the upper-right, one collapsed structure lies parallel to the focal plane and across a crack in the substrate. This microstructure responds to magnetic actuation. No magnetite aggregations are visible at the limits of optical microscopy, indicating microscale homogeneity. B) Transmission electron micrograph of crosslinked 70\% FFPDMS-NH 2 . Magnetite nanoparticles are well-dispersed throughout the material, with diameters ranging from $7-10 \mathrm{~nm}$. 

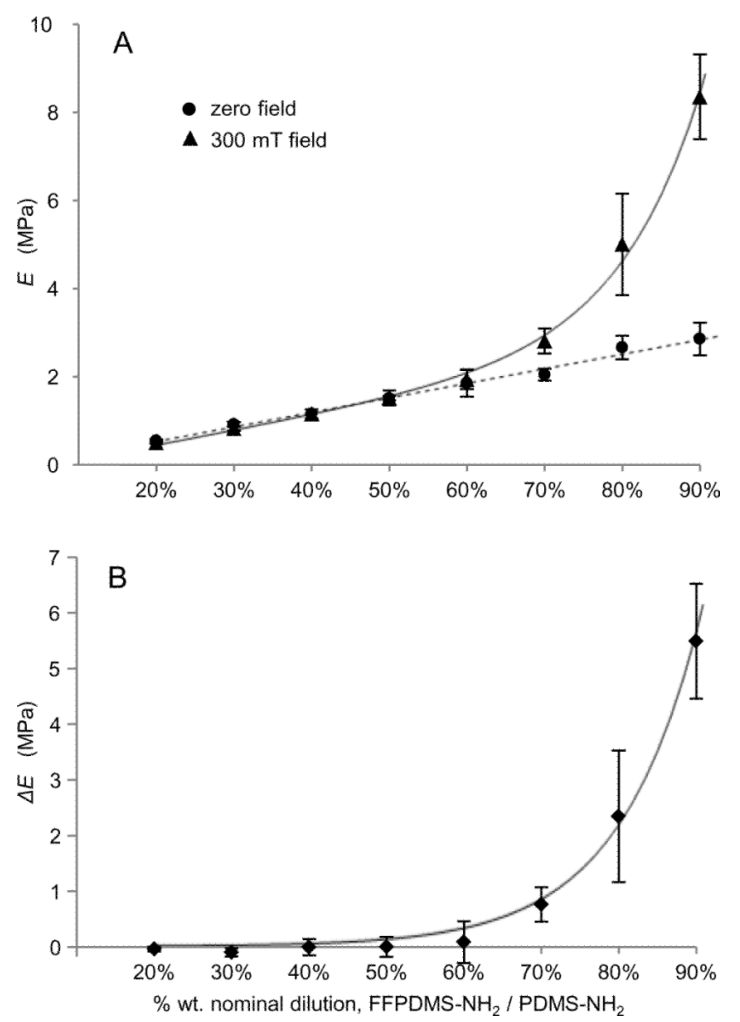

Fig. 3.

A) Young's modulus, $E$, of $20 \%-90 \%$ FFPDMS-NH 2 to $\mathrm{PDMS}_{-} \mathrm{NH}_{2}$ dilutions. Circles indicate the moduli in the absence of a magnetic field; the dotted line is a linear fit to guide the eye. Triangles indicate moduli in a $300 \mathrm{mT}$ field oriented parallel to the direction of compression; the solid curve is a fit of the form $\Delta E(\varphi)=\alpha \exp ^{\beta \varphi}+\gamma \varphi+\delta$, where $\alpha=1100$ $\mathrm{Pa}, \beta=9.5, \gamma=3.3 \times 10^{6} \mathrm{~Pa}$, and $\delta=-220,000 \mathrm{~Pa}$. B) The difference in modulus, $\Delta E$, due to the effect of the applied magnetic field. The curve is a fit of the form $\Delta E(\varphi)=\alpha \exp \beta \varphi$, where $\alpha=1100 \mathrm{~Pa}$ and $\beta=9.5$. 


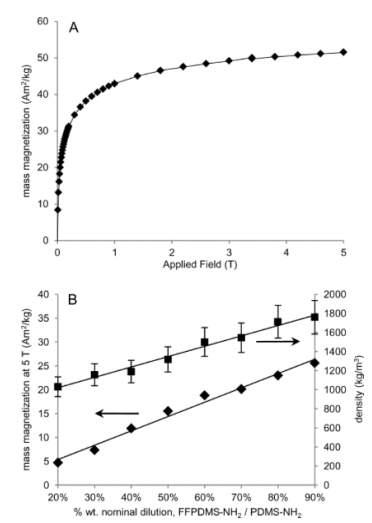

Fig. 4.

A) Magnetization curve of magnetite nanoparticles. B) Saturation magnetization (squares) and mass density (diamonds) of 20\% - 90\% FFPDMS- $\mathrm{NH}_{2}$ to PDMS-NH 2 dilutions. Saturation magnetization was measured at $5 \mathrm{~T}$ by SQUID magnetometry. 


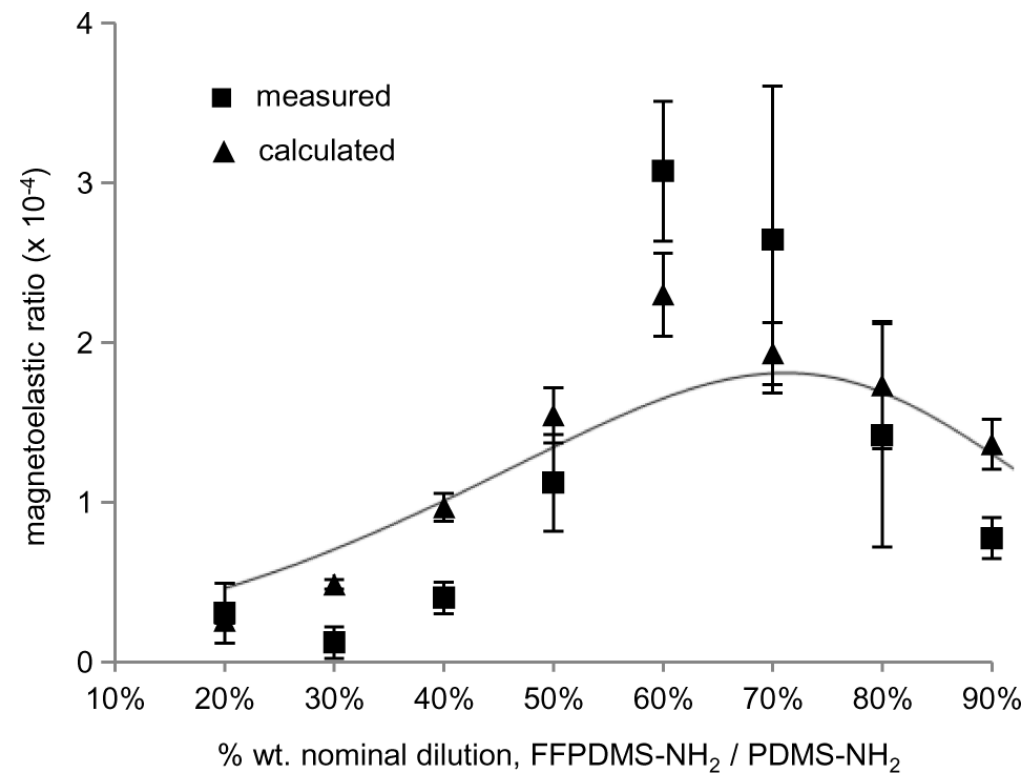

Fig. 5.

Magnetoelastic ratios of $20 \%-90 \%$ FFPDMS-NH 2 to $\mathrm{PDMS}_{2} \mathrm{NH}_{2}$ dilutions. Triangles indicate magnetoelastic ratios calculated from modulus and magnetization data obtained for each material. Squares indicate magnetoelastic ratios calculated from microactuator bending experiments. The curve is calculated according to Equation 2 using the fit to the modulus data in Figure 3A and linear fits to the magnetization and density shown in Figure 4B. 


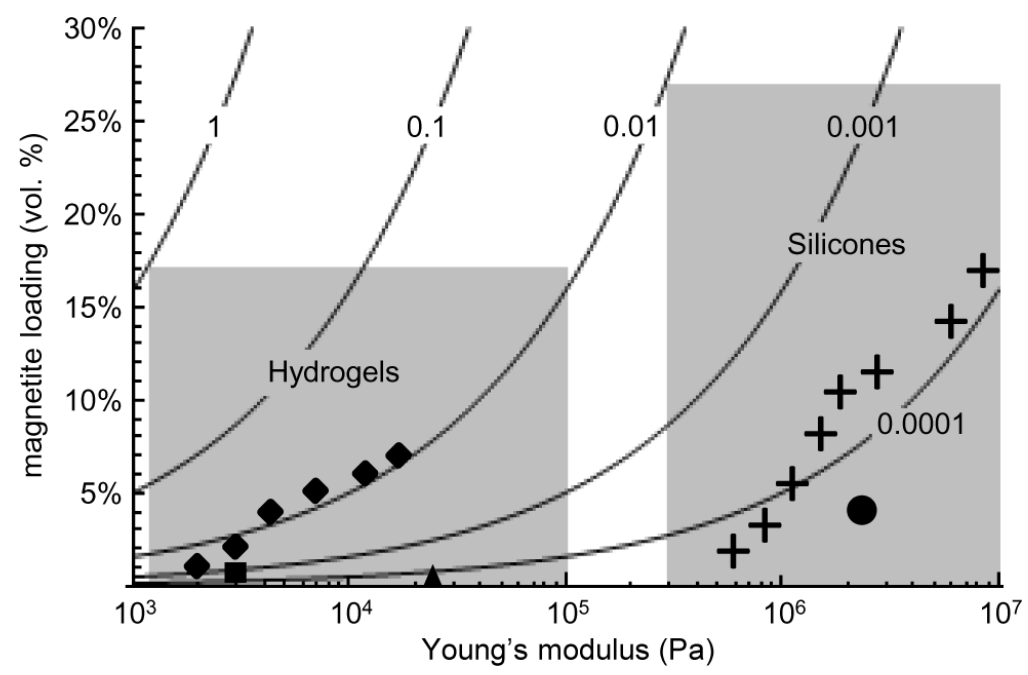

Fig. 6.

Magnetoelastic ratios for this work and reports from literature. Solid curves represent a constant magnetoelastic ratio as a function of magnetite loading and Young's modulus. Superimposed on the plot are instances of reported magnetic elastomers. Crosses: FFPDMS$\mathrm{NH}_{2}(20 \%-90 \%$ FFPDMS-NH 2$)$ presented in this work. Diamonds: maghemite / polyacrylamide (Galicia et al. 2003)[11]; Square: magnetite / polyvinyl alcohol (Barsi et al. 1996)[14]; Triangle: M-300 / polyvinyl alcohol (Collin et al. 2003)[15]; Circle: maghemite / poly(dimethyl siloxane) (Evans et al. 2007)[8]; Shaded areas indicate regions potentially accessible by hydrogel-based and silicone based magnetic elastomers, respectively. 


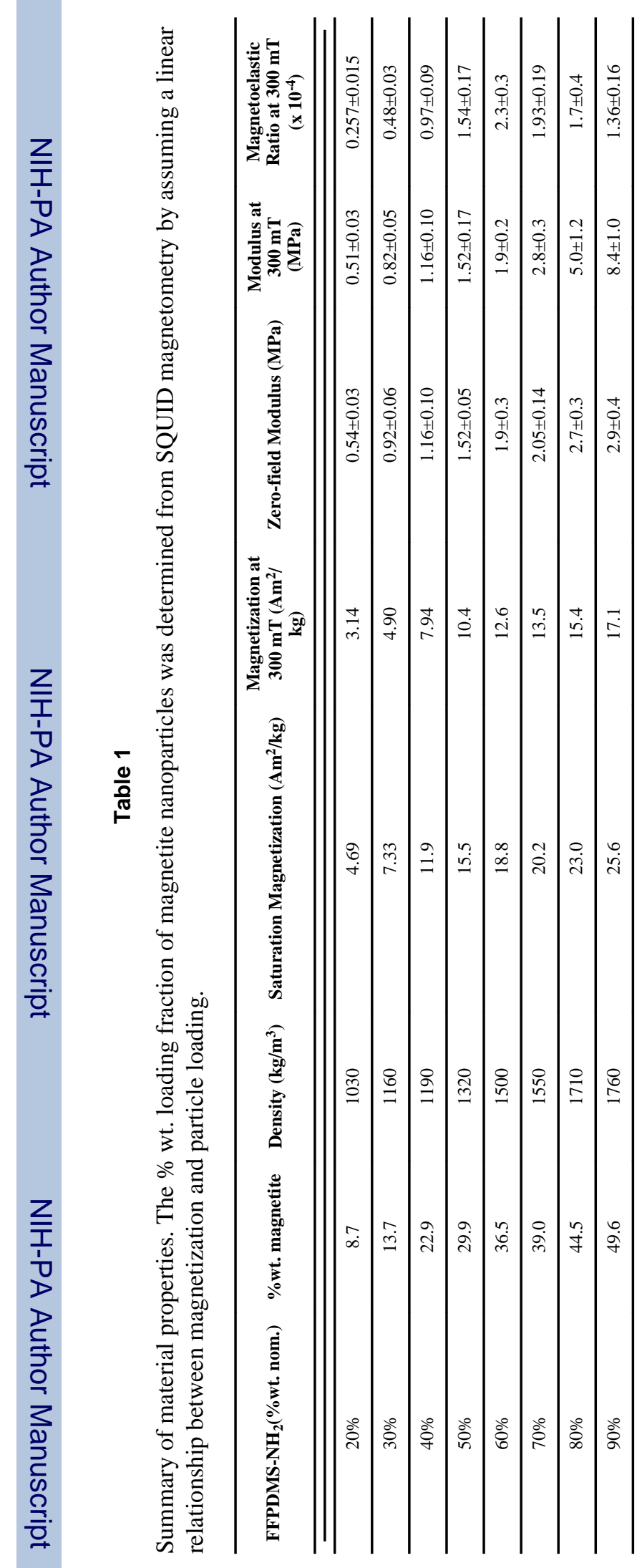

J Magn Magn Mater. Author manuscript; available in PMC 2013 February 1. 